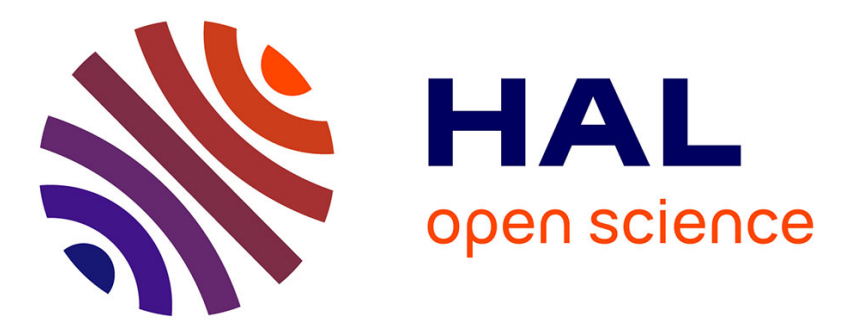

\title{
A Razumikhin approach for the incremental stability of delayed nonlinear systems
}

Antoine Chaillet, Alexander Pogromsky, Björn Rüffer

\section{To cite this version:}

Antoine Chaillet, Alexander Pogromsky, Björn Rüffer. A Razumikhin approach for the incremental stability of delayed nonlinear systems. 52nd IEEE Conference on Decision and Control CDC 2013, Dec 2013, Florence, Italy. pp.1-8, 10.1109/cdc.2013.6760110 . hal-00855656

\section{HAL Id: hal-00855656 \\ https://hal-centralesupelec.archives-ouvertes.fr/hal-00855656}

Submitted on 29 Aug 2013

HAL is a multi-disciplinary open access archive for the deposit and dissemination of scientific research documents, whether they are published or not. The documents may come from teaching and research institutions in France or abroad, or from public or private research centers.
L'archive ouverte pluridisciplinaire HAL, est destinée au dépôt et à la diffusion de documents scientifiques de niveau recherche, publiés ou non, émanant des établissements d'enseignement et de recherche français ou étrangers, des laboratoires publics ou privés. 


\title{
A Razumikhin approach for the incremental stability of delayed nonlinear systems*
}

\author{
Antoine Chaillet $^{1}$, Alexander Yu. Pogromsky ${ }^{2,3}$, Björn S. Rüffer ${ }^{4}$
}

\begin{abstract}
This paper provides sufficient conditions for the incremental stability of time-delayed nonlinear systems. It relies on the Razumikhin-Lyapunov approach, which consists in invoking small-gain arguments by treating the delayed state as a feedback perturbation. The results are valid for multiple delays, as well as bounded time-varying delays. We provide conditions under which the limit solution of a time-delayed nonlinear system in response to a periodic (resp. constant) input is itself periodic and of the same period (resp. constant). As an illustration, a specific focus is given on a class of delayed Lur'e systems.
\end{abstract}

\section{INTRODUCTION}

Lyapunov methods to study the asymptotic stability of an equilibrium or a specific set have been the subject of a wide literature. Many applications require a stronger property, namely that close initial conditions generate close solutions, that eventually converge to one another. This property is the key purpose of the closely related notions of convergence [6], contraction [17] and incremental stability [3]. The intimate correlation between them has been underlined in the recent work [26]. These three properties have proved useful in several analysis and control applications; examples include biological networks [27], synchronization of oscillators [5], observer design [1], output regulation [20], cooperative control of robots [4], and symbolic modeling [23].

Several methods can be used to establish these properties in practice. The historical ones rely on the study of the Jacobian of the vector field [6], thus performing a local analysis to guarantee global properties. This approach has been extended to more general metrics in [17]. Other methods rely on the use of an incremental Lyapunov function which vanishes whenever the two considered states coincide [3], [26]. A link between these two methodological families has recently been made in [8], using the notion of Finsler-Lyapunov functions.

Nonetheless, in presence of delays, only few results exist to establish incremental properties. A notable exception is [23], which makes use of Lyapunov-Krasovskii functionals to establish incremental input-to-state stability $(\delta$-ISS) of systems with possibly time-varying delays. In view of the pervasive presence of delays in economy, biology, neuroscience, communication or transportation models [18], we here present a sufficient

\footnotetext{
${ }^{1}$ L2S - Univ. Paris Sud 11 - Supélec, 3, rue Joliot-Curie, 91192 Gif-surYvette, France, antoine. chailletesupelec.fr.

${ }^{2}$ Dept. of Mechanical Engineering, Eindhoven University of Technology, Eindhoven, The Netherlands, a.pogromsky@tue.nl.

${ }^{3}$ Dept. of Control Systems and Informatics, Saint-Petersburg National Research University of Information Technologies Mechanics and Optics.

${ }^{4}$ Signal and System Theory Group, Faculty of Electrical Engineering, Computer Science, and Mathematics, Universität Paderborn, Germany, bjoern. ruefferduni-paderborn. de.

*The work of A. Chaillet has received funding from the European Union Seventh Framework Program [FP7/2007-2013] under grant agreement n257462 HYCON2 Network of excellence.
}

condition for a nonlinear time-delayed system to be uniformly incrementally stable, thus complementing the existing works on $\delta$-ISS by imposing that the convergence rate be uniform in the applied input. Our results rely on the RazumikhinLyapunov approach [25], [11], which consists in treating the delayed state as a feedback perturbation and in deriving smallgain conditions for the overall system to preserve stability. Following the steps of [29], [30], we provide a small-gain condition on the associated incremental Lyapunov function for a generic time-delay nonlinear system to be incrementally stable.

The considered class of systems allows the vector field to be time-varying, thus permitting the study of systems with exogenous inputs. We invoke the fact that the notions of convergence and incremental stability are equivalent for systems involving in a compact set [26] to provide conditions under which the delayed dynamics asymptotically tends to a limit solution which is $T$-periodic provided that the applied input is itself $T$-periodic. Similarly, these conditions ensure that the response to a constant input also tends to a constant value. These features pave the way towards a nonlinear frequency analysis, with the notion of nonlinear Bode plot [21]. Our results are valid for a wide class of nonlinear time-delayed systems, and allow for multiple and time-varying delays, as long as they are upper-bounded by a known constant.

Motivated by neuroscience applications, we then focus on a class of Lur'e systems with multiple delays. We provide explicit sector-bounded conditions on the nonlinearity to guarantee incremental stability of the system despite the presence of delays. The obtained conditions are delay-independent, meaning that the small-gain conditions ensure incremental stability regardless of the length of the delays involved.

The paper is organized as follows. The necessary definitions and the extension of the Razumikhin-Lyapunov approach to incremental stability are presented in Section II. The result concerning the $T$-periodic response to a $T$-periodic input is provided in Section III. The analysis of Lur'e systems with multiple delays is given in Section IV. All proofs are proposed in Section V. Some conclusions and perspectives are provided in Section VI.

Notation. Given $x \in \mathbb{R}^{n}$ and $\varepsilon \geq 0, \mathcal{B}(x, \varepsilon):=$ $\left\{z \in \mathbb{R}^{n}:|x-z| \leq \varepsilon\right\}$, where $|\cdot|$ denotes the Euclidean norm. Given two real numbers $a<b, \mathcal{C}([a, b])$ denotes the set of all continuous functions from $[a ; b]$ to $\mathbb{R}$. This notation extends to any interval of $\mathbb{R}$. Given any $\theta \geq 0$, the set $\mathcal{C}([-\theta,+\infty))$ is equipped with the $L_{\infty}$ norm: for each $x \in \mathcal{C}([-\theta,+\infty))^{n}$ and any $-\theta \leq t_{1}<t_{2}$, $\|x\|_{\left[t_{1} ; t_{2}\right]}:=\sup _{t \in\left[t_{1} ; t_{2}\right]}|x(t)|$. We will also write $\|x\|:=$ $\sup _{t \in[-\theta ;+\infty)}|x(t)|$. Given a signal $q: \mathbb{R}_{\geq-\theta} \rightarrow \mathbb{R}^{n}$ and a 
time instant $t \in \mathbb{R}_{>0},(q)_{t}$ denotes the signal defined as

$$
(q)_{t}:\left\{\begin{array}{cll}
{[-\theta ; 0]} & \rightarrow & \mathbb{R}^{n} \\
s & \mapsto & q(t+s) .
\end{array}\right.
$$

A function $\alpha: \mathbb{R}_{\geq 0} \rightarrow \mathbb{R}_{\geq 0}$ is of class $\mathcal{K}$ if it is continuous, strictly increasing and zero at zero. It is of class $\mathcal{K}_{\infty}$ if it is of class $\mathcal{K}$ and unbounded. A function $\sigma$ is of class $\mathcal{L}$ is is continuous, non-increasing, and tends to zero as its argument tends to infinity. A function $\beta: \mathbb{R}_{\geq 0} \times \mathbb{R}_{\geq 0} \rightarrow \mathbb{R}_{\geq 0}$ belongs to class $\mathcal{K} \mathcal{L}$ if, given any $t \geq 0, \beta(\cdot, \bar{t}) \in \mathcal{K}$ and, given any $s \geq 0$, $\beta(s, \cdot) \in \mathcal{L}$.

\section{A RAZUMIKHIN CONDITION FOR INCREMENTAL STABILITY}

We start by extending the Razumikhin approach to incremental stability properties. We consider delayed nonlinear systems of the form

$$
\dot{x}(t)=f\left(t, x(t),(x)_{t}\right),
$$

where $f: \mathbb{R}_{\geq 0} \times \mathbb{R}^{n} \times \mathcal{C}([-\theta ; 0])^{n} \rightarrow \mathbb{R}^{n}$. We assume that the image by $f$ of any bounded set of $\mathbb{R}_{\geq 0} \times \mathbb{R}^{n} \times \mathcal{C}([-\theta ; 0])^{n}$ is a bounded set of $\mathbb{R}^{n}$, and that $f$ is Lipschitz continuous on any compact set of $\mathbb{R}_{\geq 0} \times \mathbb{R}^{n} \times \mathcal{C}([-\theta ; 0])^{n}$.

We stress that the class of systems (1) includes systems with arbitrarily large (but bounded) multiple delays. To see this, it is sufficient to consider in the definition of $(x)_{t}$ any constant $\theta$ larger that the greatest of the delays involved. These discrete delays may be non-commensurate. Time-varying and distributed delays are also covered.

Definition 1 (Incremental stability for time-delayed systems): The system (1) is said to be uniformly incrementally stable if there exists $\beta \in \mathcal{K} \mathcal{L}$ such that, given any initial time $t_{0} \in \mathbb{R}_{\geq 0}$ and any two initial conditions $\xi, \zeta \in \mathcal{C}\left(\left[t_{0}-\theta ; t_{0}\right]\right)^{n}$, its solutions satisfy, for all $t \geq t_{0}$,

$$
\left|\varphi\left(t ; t_{0}, \xi\right)-\varphi\left(t ; t_{0}, \zeta\right)\right| \leq \beta\left(\|\xi-\zeta\|_{\left[t_{0}-\theta ; t_{0}\right]}, t-t_{0}\right) .
$$

Similarly to its non-delayed version [3], incremental stability imposes that any two trajectories of (1) eventually tend to one another, and that the maximum distance between them during transients is somewhat proportional to the magnitude of the difference between initial states. "Uniformly" is here to be understood as the fact that the convergence rate $\beta$ is independent of the considered initial time $t_{0}$. In the case when the time-dependency of (1) results from the application of an exogenous input, "uniformity" imposes that the convergence rate be independent of the applied input. Hence, uniform incremental stability should not be confused with incremental ISS ( $\delta$-ISS, [3]), which allows solutions to converge to one another up to a tolerance "proportional" to the input amplitude and for which alternative approaches involving Lyapunov-Krasovskii functionals already exist [23].

As recalled above, the Razumikhin approach to analyze the stability of systems of the form (1) consists in treating the delayed-state as a perturbation and in invoking small-gain arguments to guarantee that its influence does not compromise stability [25], [11], [29]. See also [28] for an application of this method to synchronization analysis purposes. It therefore relies on the study of the following non-delayed version of (1):

$$
\dot{x}(t)=f(t, x(t), w)
$$

where $w \in \mathcal{C}([-\theta ;+\infty))^{n}$ is an exogenous input. In the whole paper, we will refer to the solution of the delayed system (1) as $\varphi$, whereas the solution of the non-delayed system (2) will be indicated by $\phi$. The result below extends Razumikhin's approach to incremental stability.

Theorem 1: Assume that there exist a continuously differentiable function $V: \mathbb{R} \times \mathbb{R}^{n} \times \mathbb{R}^{n} \rightarrow \mathbb{R}_{\geq 0}$, class $\mathcal{K}_{\infty}$ functions $\underline{\alpha}, \bar{\alpha}$ and $\kappa$, and a class $\mathcal{K}$ function $\rho$ such that, for all $t \in \mathbb{R}_{\geq 0}$, all $x, y \in \mathbb{R}^{n}$, and all $v, w \in \mathcal{C}([-\theta ;+\infty))^{n}$,

$$
\begin{gathered}
\underline{\alpha}(|x-y|) \leq V(t, x, y) \leq \bar{\alpha}(|x-y|) \\
V(t, x, y) \geq \kappa(\|v-w\|) \quad \Rightarrow \quad \dot{V} \leq-\rho(|x-y|),
\end{gathered}
$$

where

$$
\dot{V}:=\frac{\partial V}{\partial t}+\frac{\partial V}{\partial x} f(t, x, v)+\frac{\partial V}{\partial y} f(t, y, w) .
$$

Assume also that, for each $t_{0} \in \mathbb{R}_{\geq 0}$, (1) admits at least one solution defined over $\mathbb{R}_{\geq t_{0}}$ and that the following small-gain condition holds:

$$
\underline{\alpha}^{-1} \circ \kappa(s)<s, \quad \forall s \in \mathbb{R}_{>0} .
$$

Then the delayed system (1) is uniformly incrementally stable.

This result basically states that if the non-delayed system (2) is incrementally input-to-state stable [3] and the nonlinear small-gain condition (5) holds, then the delayed system (1) is uniformly incrementally stable, regardless of the nature and size of the delays involved. The advantage of this approach lies in the simplicity of its application, as only tools from finitedimensional systems are needed. Nonetheless, it does not allow to derive delay-dependent conditions for incremental stability and requires strong robustness properties of (2).

Theorem 1 is an immediate consequence of the following two lemmas. The first one states that an explicit estimate of the norm between two solutions can be obtained for the nondelayed system (2) based on the knowledge of an incremental Lyapunov function. This estimate takes the form of an upper bound involving a vanishing transient function whose amplitude is "proportional" to the difference between the considered initial conditions and a term involving the amplitude of the difference between the applied inputs.

Lemma 1: Assume that there exists a continuously differentiable $V: \mathbb{R}_{\geq 0} \times \mathbb{R}^{n} \times \mathbb{R}^{n}$ satisfying, for all $t \in \mathbb{R}_{\geq 0}$, all $x, y \in \mathbb{R}^{n}$ and all $v, w \in \mathcal{C}([-\theta ;+\infty))^{n}$,

$$
\begin{gathered}
\underline{\alpha}(|x-y|) \leq V(t, x, y) \leq \bar{\alpha}(|x-y|) \\
V(t, x, y) \geq \kappa(\|v-w\|) \quad \Rightarrow \quad \dot{V} \leq-\rho(|x-y|),
\end{gathered}
$$

with $\underline{\alpha}, \bar{\alpha}, \kappa \in \mathcal{K}_{\infty}$ and $\rho \in \mathcal{K}$. Then there exists $\beta \in \mathcal{K} \mathcal{L}$ such that the solutions of (2) satisfy, for all $t \geq t_{0} \geq 0$, all $x_{0}, y_{0} \in \mathbb{R}^{n}$ and all $v, w \in \mathcal{C}([-\theta ;+\infty))^{n}$,

$$
\begin{aligned}
& \underline{\alpha}\left(\left|\phi\left(t ; t_{0}, x_{0}, v\right)-\phi\left(t ; t_{0}, y_{0}, w\right)\right|\right) \leq \\
& \max \left\{\beta\left(\left|x_{0}-y_{0}\right|, t-t_{0}\right) ; \kappa\left(\|v-w\|_{\left[t_{0} ;+\infty\right)}\right)\right\} .
\end{aligned}
$$

For the sake of completeness, we give the proof of this result in Section V-A. However, we stress that its only contribution with respect to similar statements existing in the literature, including non-incremental or time-invariant versions, is to make an explicit link between the lower bound $\underline{\alpha}$ on $V$ and the supply 
rate $\kappa$ (cf. (6) and (7)), and the functions involved in the state estimate (8).

The second lemma is the key step in the proof of Theorem 1. It links the incremental properties of the delayed system (1) and the non-delayed system (2).

Lemma 2: Assume that there exists $\beta \in \mathcal{K} \mathcal{L}$ and $\alpha, \kappa \in$ $\mathcal{K}_{\infty}$ such that, for all $x_{0}, y_{0} \in \mathbb{R}^{n}$, all $t_{0} \in \mathbb{R}_{\geq 0}$ and all $v, w \in$ $\mathcal{C}([-\theta ;+\infty))^{n}$, the solutions of the non-delayed system (2) satisfy, for all $t \geq t_{0}$,

$$
\begin{gathered}
\alpha\left(\left|\phi\left(t ; t_{0}, x_{0}, v\right)-\phi\left(t ; t_{0}, y_{0}, w\right)\right|\right) \leq \\
\max \left\{\beta\left(\left|x_{0}-y_{0}\right|, t-t_{0}\right) ; \kappa\left(\|v-w\|_{\left[t_{0} ;+\infty\right)}\right)\right\} .
\end{gathered}
$$

Assume further that

$$
\alpha^{-1} \circ \kappa(s)<s, \quad \forall s \in \mathbb{R}_{>0} .
$$

Suppose finally that (1) admits at least one solution defined over $\mathbb{R}_{\geq t_{0}}$. Then the delayed system (1) is forward complete and there exists $\hat{\beta} \in \mathcal{K} \mathcal{L}$ such that its solutions satisfy, for all $\xi, \zeta \in \mathcal{C}\left(\left[t_{0}-\theta ; t_{0}\right]\right)^{n}$ and all $t \geq t_{0}$,

$$
\left|\varphi\left(t ; t_{0}, \xi\right)-\varphi\left(t ; t_{0}, \zeta\right)\right| \leq \hat{\beta}\left(\|\xi-\zeta\|_{\left[t_{0}-\theta ; t_{0}\right]}, t-t_{0}\right) .
$$

This result is an extension of [30, Theorem 1] to incremental properties. Its proof is provided in Section V-B.

\section{RESPONSE TO PERIODIC OR CONSTANT INPUTS}

As recently stressed in [26], a tight link exists between incremental stability and the notion of convergence. The latter, based on the original ideas of Pliss [22] and Demidovich [6], ensures the existence of a bounded "steady-state" solution to which all other solutions asymptotically converge: see [20] for details. This notion can be straightforwardly extended to delayed systems as follows.

Definition 2 (Convergence for time-delayed systems): The delayed system (1) is said to be uniformly convergent if all its solutions $\varphi\left(\cdot ; t_{0}, \xi\right)$ exist over $\left[t_{0} ;+\infty\right)$ for all $t_{0} \in \mathbb{R}$ and all $\xi \in \mathcal{C}\left(\left[t_{0}-\theta ; t_{0}\right]\right)^{n}$ and there exist a function $\beta \in \mathcal{K} \mathcal{L}$ and a unique solution $\bar{x}: \mathbb{R} \rightarrow \mathbb{R}^{n}$, defined and bounded over $\mathbb{R}$, such that, for all $t_{0} \in \mathbb{R}$ and all $\xi \in \mathcal{C}\left(\left[t_{0}-\theta ; t_{0}\right]\right)^{n}$,

$$
\left|\varphi\left(t ; t_{0}, \xi\right)-\bar{x}(t)\right| \leq \beta\left(\|\xi-x\|_{\left[t_{0}-\theta ; t_{0}\right]}, t-t_{0}\right), \quad \forall t \geq t_{0} .
$$

As already stressed, the time-dependency of (1) may arise from the application of an exogenous input. In that case, a noteworthy feature of uniformly convergent dynamics is the property that the response to any periodic input asymptotically tends to a periodic solution of the same period. Similarly, if the input is constant, then the state of a convergent system asymptotically converges to a constant value. This feature, shared by all stable linear time-invariant systems, is far from being common among nonlinear systems. It opens the door to an extension of frequency response analysis, with the notion of nonlinear Bode plot introduced in [21].

Convergence and incremental stability happen to be equivalent if solutions evolve on a compact set. This fact has been recently proven in [26] for non-delayed systems and can be readily adapted to time-delayed system. The proof is presented in Section V-C.

Proposition 1: Assume that the solutions of the timedelayed system (1) all converge to a compact forward invariant set of $\mathbb{R}^{n}$, then (1) is uniformly incrementally stable if and only if it is uniformly convergent.

Based on this observation, the following statement provides conditions under which the limit response of a uniformly incrementally stable system to a periodic input is itself periodic of the same period. Its proof is provided in Section V-D.

Proposition 2: Assume that the delayed nonlinear system (1) is uniformly incrementally stable and that the vector field $f(\cdot, x, y)$ is periodic of period $T \geq 0$ for any fixed $x \in \mathbb{R}^{n}$ and any fixed $y \in \mathcal{C}([-\theta ;+\infty))^{n}$. Assume further that there exists a compact set $M \subset \mathbb{R}^{n}$ forward invariant for (1). Then there exists a $T$-periodic signal $\bar{x} \in \mathcal{C}([-\theta ;+\infty))^{n}$ and a $\mathcal{K} \mathcal{L}$ function $\beta$ such that, for all $t_{0} \in \mathbb{R}_{\geq 0}$ and all $\xi \in \mathcal{C}\left(\left[t_{0}-\theta ; t_{0}\right]\right)^{n}$, the solutions of (1) satisfy

$\left|\varphi\left(t ; t_{0}, \xi\right)-\bar{x}(t)\right| \leq \beta\left(\|\xi-\bar{x}\|_{\left[t_{0}-\theta ; t_{0}\right]}, t-t_{0}\right), \quad \forall t \geq t_{0}$.

In particular, if $f$ is independent of $t$, then the globally asymptotically stable steady-state solution $\bar{x}$ is constant.

Since the time-dependency of the vector field $f$ typically arises from the application of an exogenous signal, the above result basically states that the limit solution of a uniformly incrementally stable system evolving on a forward invariant set (hence, convergent) in response to any $T$-periodic (resp. constant) input is itself $T$-periodic (resp. constant). This feature is illustrated by the example presented next.

\section{Application to LUR'E SYSTEMS With MULTIPLE DELAYS}

One of the motivations for the present study was the possibility to analyze the generation of pathological oscillations within a network of interconnected neuronal populations in the context of Parkinson's disease. More precisely, it is well established [12] that the motor symptoms of Parkinson's disease are directly linked to the strength of beta oscillations $(13-30 \mathrm{~Hz})$ in the subthalamic nucleus (STN), which is a neuronal structure interconnected with other brain zones including global pallidus pars externa (GPe), cortex, striatum, and possibly pedunculopontine nucleus (PPN). Recent works [14], [19], [10] have demonstrated that the interconnection strength and the propagation delays between these populations (mainly, STN, GPe and PPN) may generate cerebral oscillations. However, the methods used in those reference did not allow to tackle simultaneously both nonlinearities and delays.

The following result is a first step in that direction as it establishes conditions for the incremental stability of a wider class of systems, namely:

$$
\dot{x}=A x+g\left(t,(x)_{t}\right)
$$

where $A \in \mathbb{R}^{n \times n}$ and the nonlinearity $g=\left(g_{1}, \ldots, g_{n}\right)^{T}$ : $\mathbb{R}_{\geq 0} \times \mathcal{C}([-\theta ;+\infty))^{n}$ is of the form

$$
g_{i}\left(t,(x)_{t}\right)=S_{i}\left(u_{i}(t)+\sum_{j=1}^{n} c_{i j} x_{j}\left(t-\delta_{i j}\right)\right),
$$

where $c_{i j} \in \mathbb{R}$ and $\delta_{i j} \in[0 ; \theta]$ for all $i, j \in\{1, \ldots, n\}$, for some $\theta>0$. This class of systems indeed encompasses the firing-rate models used in [14], [19], [10].

We stress that, for this system, the only explicit timedependency results from the action of the input $u=$ $\left(u_{1}, \ldots, u_{n}\right)^{T}$. 
Systems of the form (12)-(13) are a delayed version of Lur'e systems, which have been the object of a wide literature: see for instance [2], [7], [16]. In particular, a first study of the incremental properties of Lur'e systems (in the absence of delays) was provided in [31]. See [15] for a study of Lur'e systems using ISS small-gain arguments. See also [24] for an absolute stability criterion of delayed linear systems affected by a non-delayed nonlinearity, and [13] for an LMI-based analysis allowing for delays in the nonlinearity. This section provides conditions under which (12)-(13) is incrementally stable and asymptotically responds to a periodic (resp. constant) input $u$ with a periodic (resp. constant) state. It relies on the following two assumptions. The first one requires the matrix $A$ to be Hurwitz.

Assumption 1: There exist two symmetric positive definite matrices $P, Q \in \mathbb{R}^{n \times n}$ such that $A^{T} P+P A \leq-Q$.

The second one imposes a sector bound on the nonlinearity $g$.

Assumption 2: For each $i \in \mathbb{N}_{\leq n}$, the function $S_{i}$ is globally Lipschitz with Lipschitz constant $\sigma_{i} \in \mathbb{R}_{\geq 0}$, that is:

$$
\left|S_{i}\left(\nu_{1}\right)-S_{i}\left(\nu_{2}\right)\right| \leq \sigma_{i}\left|\nu_{1}-\nu_{2}\right|, \quad \forall \nu_{1}, \nu_{2} \in \mathbb{R} .
$$

We stress that, even though the results of Sections II and III are valid for locally Lispchitz dynamics, the functions $S_{i}$ are here assumed to be globally Lipschitz; the values of the Lipschitz constants $\sigma_{i}$ are indeed instrumental for the incremental stability of (12) (see condition (15) below).

The main result of this section is summarized by the following statement.

Proposition 3: Under Assumptions 1 and 2, let

$$
p_{m}:=\min _{|x|=1}|P x|, \quad p_{M}:=\max _{|x|=1}|P x|,
$$

and let $\lambda_{0} \geq 0$ denote the smallest solution of the algebraic equation

$$
\operatorname{det}(P-\lambda Q)=0
$$

If the following condition is satisfied:

$$
\sqrt{\sum_{i=1}^{n}\left(\sigma_{i} \sum_{j=1}^{n}\left|c_{i j}\right|\right)^{2}}<\frac{p_{m}}{2 p_{M} \lambda_{0}}
$$

then the system (12)-(13) is uniformly incrementally stable for all bounded inputs $u \in \mathcal{C}([0 ;+\infty))^{n}$. Furthermore, if the functions $S_{i}, i \in\{1, \ldots, n\}$, are bounded then, for any periodic input $u \in \mathcal{C}([0 ;+\infty))^{n}$, all its solutions tend to a periodic solution of same period. Finally, if the input $u$ is constant, then (12)-(13) admits a unique equilibrium which is globally asymptotically stable.

We stress that the conservatism of condition (15) may strongly depend on the choice of the matrices $P$ and $Q$. Numerical investigations may be used to tighten this bound.

Remark 1: Condition (15) can be replaced by

$$
\sqrt{\sum_{i=1}^{n}\left(\sigma_{i} \sum_{j=1}^{n}\left|c_{i j}\right|\right)^{2}}<\frac{p_{m} q_{m}}{2 p_{M}^{2}}
$$

where $q_{m}:=\min _{|x|=1}|Q x|$. This condition is more conservative than (15), but does not require to solve the algebraic equation (14).
The detailed application of the present work to the brain oscillations analysis in Parkinson's disease will be the subject of a future work, based on theoretical advances presented here. They are expected to provide conditions for the generation of pathological oscillations by taking into account both delays and nonlinearities. The possibility to exploit nonlinear Bode plots [21] will constitute a particularly relevant tool in order to estimate the frequency spectrum that is preferentially amplified by the network of neuronal populations, thus characterizing the nature of the generated oscillations.

\section{PROOFS}

\section{A. Proof of Lemma 1.}

Given any $x_{0}, y_{0} \in \mathbb{R}^{n}$, any $t_{0} \in \mathbb{R}_{\geq 0}$, and any $v, w \in \mathcal{C}([-\theta ;+\infty))^{n}$, let $\mathcal{V}(\cdot) \quad$ $V\left(\cdot, \phi\left(\cdot ; t_{0}, x_{0}, v\right), \phi\left(\cdot ; t_{0}, y_{0}, w\right)\right)$ everywhere it exists. Note that the regularity assumptions made on the vector field $f$ guarantee the existence of $\mathcal{V}$ on an open interval containing $t_{0}$ [11]. Let also $a:=\kappa\left(\|u-v\|_{\left[t_{0} ; \infty\right)}\right)$. Then (7) implies that

$$
\mathcal{V}(t) \geq a \quad \Rightarrow \quad \dot{\mathcal{V}}(t) \leq-\rho(\mathcal{V}(t))
$$

This inequality guarantees the boundedness of $\mathcal{V}$, and consequently its existence over $\left[t_{0} ; \infty\right)$. It also ensures that, given any $t^{*} \geq t_{0}$,

$$
\mathcal{V}\left(t^{*}\right) \leq a \quad \Rightarrow \quad \mathcal{V}(t) \leq a, \quad \forall t \geq t^{*}
$$

Let $\tau:=\inf \left\{t \geq t_{0}: \mathcal{V}(t) \leq a\right\} \in \mathbb{R}_{\geq t_{0}} \cup\{\infty\}$. Then it holds that $\dot{\mathcal{V}}(t) \leq-\rho(\mathcal{V}(t))$ for all $t \in\left[t_{0} ; \tau\right]$. The comparison lemma together with [16, Lemma 4.4] then ensure the existence of a $\mathcal{K} \mathcal{L}$ function $\beta_{0}$ such that

$$
\mathcal{V}(t) \leq \beta_{0}\left(\mathcal{V}\left(t_{0}\right), t-t_{0}\right) ; \quad \forall t \in\left[t_{0} ; \tau\right)
$$

Noticing that, by continuity of solutions, $\mathcal{V}(\tau)=a$, we get from (17) that

$$
\mathcal{V}(t) \leq a, \quad \forall t \geq \tau .
$$

Combining the last two bounds and recording the definition of $a$ yields, for all $t \geq t_{0}$,

$$
\mathcal{V}(t) \leq \max \left\{\beta_{0}\left(\mathcal{V}\left(t_{0}\right), t-t_{0}\right) ; \kappa\left(\|u-v\|_{\left[t_{0} ; \infty\right)}\right)\right\} .
$$

Using (6), we finally obtain (8) by picking $\beta$ as the $\mathcal{K} \mathcal{L}$ function defined by $\beta(s, t):=\beta_{0}(\bar{\alpha}(s), t)$ for all $s, t \geq 0$.

\section{B. Proof of Lemma 2.}

We recall that the symbol $\varphi$ is used to denote the solutions of the delayed system (1), while the solutions of the non-delayed system (2) are denoted by $\phi$. First notice that, due to causality, (9) can be rewritten as

$$
\begin{gathered}
\alpha\left(\left|\phi\left(t ; t_{0}, x_{0}, v\right)-\phi\left(t ; t_{0}, y_{0}, w\right)\right|\right) \leq \\
\max \left\{\beta\left(\left|x_{0}-y_{0}\right|, t-t_{0}\right) ; \kappa\left(\|v-w\|_{\left[t_{0} ; t\right]}\right)\right\} .
\end{gathered}
$$

Consider any $t_{0} \in \mathbb{R}_{\geq 0}$ and any $\xi, \zeta \in \mathcal{C}\left(\left[t_{0}-\theta ; t_{0}\right]\right)^{n}$. Let $T_{1}\left(t_{0}, \xi\right), T_{2}\left(t_{0}, \zeta\right) \in \mathbb{R}_{\geq 0} \cup\{\infty\}$ be such that $\left[t_{0} ; t_{0}+T_{1}\right]$ and $\left[t_{0} ; t_{0}+T_{2}\right]$ are the maximal intervals of existence of $\varphi\left(\cdot ; t_{0}, \xi\right)$ and $\varphi\left(\cdot ; t_{0}, \zeta\right)$, and let $\bar{T}:=\min \left\{T_{1}, T_{2}\right\}$. By classical considerations on the existence of solutions of timedelayed systems (cf. [11, Theorem 2.1]), it then holds that $\bar{T} \in$ $\mathbb{R}_{>0} \cup\{\infty\}$. For notation compactness, let $\varphi_{\xi}(\cdot):=\varphi\left(\cdot ; t_{0}, \xi\right)$ and $\varphi_{\zeta}(\cdot):=\varphi\left(\cdot ; t_{0}, \zeta\right)$ over their respective maximal intervals of existence. 
By picking $x_{0}=\xi\left(t_{0}\right)$ and $y_{0}=\zeta\left(t_{0}\right)$, the solutions of (1) and (2) satisfy, for all $t \in\left[t_{0} ; t_{0}+\bar{T}\right]$,

$$
\begin{aligned}
& \varphi_{\xi}(t)=\phi\left(t ; t_{0}, x_{0},\left(\varphi_{\xi}\right)_{t}\right) \\
& \varphi_{\zeta}(t)=\phi\left(t ; t_{0}, y_{0},\left(\varphi_{\zeta}\right)_{t}\right) .
\end{aligned}
$$

In view of (18), we then have that, for all $t \in\left[t_{0} ; t_{0}+\bar{T}\right]$,

$$
\begin{array}{r}
\alpha\left(\left|\varphi_{\xi}(t)-\varphi_{\zeta}(t)\right|\right) \leq \max \left\{\beta\left(\left|\xi\left(t_{0}\right)-\zeta\left(t_{0}\right)\right|, t-t_{0}\right) ;\right. \\
\left.\kappa\left(\left\|\left(\varphi_{\xi}\right)_{t}-\left(\varphi_{\zeta}\right)_{t}\right\|_{\left[t_{0} ; t\right]}\right)\right\},
\end{array}
$$

which yields

$$
\begin{array}{r}
\alpha\left(\left|\varphi_{\xi}(t)-\varphi_{\zeta}(t)\right|\right) \leq \max \left\{\beta\left(\left|\xi\left(t_{0}\right)-\zeta\left(t_{0}\right)\right|, t-t_{0}\right) ;\right. \\
\left.\kappa\left(\left\|\varphi_{\xi}-\varphi_{\zeta}\right\|_{\left[t_{0}-\theta ; t\right]}\right)\right\} .
\end{array}
$$

$\alpha$ being a class $\mathcal{K}_{\infty}$ function, this ensures that

$$
\begin{aligned}
\left\|\varphi_{\xi}-\varphi_{\zeta}\right\|_{\left[t_{0} ; t_{0}+\bar{T}\right]} \leq \max \{ & \alpha^{-1} \circ \beta\left(\left|\xi\left(t_{0}\right)-\zeta\left(t_{0}\right)\right|, 0\right) ; \\
& \left.\alpha^{-1} \circ \kappa\left(\left\|\varphi_{\xi}-\varphi_{\zeta}\right\|_{\left[t_{0}-\theta ; t\right]}\right)\right\} .
\end{aligned}
$$

Recalling that $\varphi_{\xi}(t)=\xi(t)$ and $\varphi_{\zeta}(t)=\zeta(t)$ for all $t \in$ $\left[t_{0}-\theta ; t_{0}\right]$, and taking the supremum of the above bound over $\left[t_{0} ; t\right]$, we get that

$$
\begin{aligned}
& \left\|\varphi_{\xi}-\varphi_{\zeta}\right\|_{\left[t_{0} ; t\right]} \leq \max \left\{\alpha^{-1} \circ \beta\left(\left|\xi\left(t_{0}\right)-\zeta\left(t_{0}\right)\right|, 0\right) ;\right. \\
& \left.\quad \alpha^{-1} \circ \kappa\left(\|\xi-\zeta\|_{\left[t_{0}-\theta ; t_{0}\right]}\right) ; \alpha^{-1} \circ \kappa\left(\left\|\varphi_{\xi}-\varphi_{\zeta}\right\|_{\left[t_{0} ; t\right]}\right)\right\} .
\end{aligned}
$$

By the small gain condition (11), this necessarily implies that

$$
\begin{aligned}
& \left\|\varphi_{\xi}-\varphi_{\zeta}\right\|_{\left[t_{0} ; t\right]} \leq \max \left\{\alpha^{-1} \circ \beta\left(\left|\xi\left(t_{0}\right)-\zeta\left(t_{0}\right)\right|, 0\right) ;\right. \\
& \left.\alpha^{-1} \circ \kappa\left(\|\xi-\zeta\|_{\left[t_{0}-\theta ; t_{0}\right]}\right)\right\}
\end{aligned}
$$

and we obtain that

$$
\left\|\varphi_{\xi}-\varphi_{\zeta}\right\|_{\left[t_{0} ; t\right]} \leq \sigma_{0}\left(\|\xi-\zeta\|_{\left[t_{0}-\theta ; t_{0}\right]}\right)
$$

where $\sigma_{0}$ denotes the class $\mathcal{K}$ function defined as $\sigma_{0}(s):=$ $\max \left\{\alpha^{-1} \circ \beta(s, 0) ; \alpha^{-1} \circ \kappa(s)\right\}$.

By assumption, at least one trajectory of (1) exists at all times $t \geq t_{0}$. Applying the above bound by considering this particular forward complete solution and any other solution of the system ensures that all solutions of (1) exist at all times $t \geq t_{0}$, meaning that $\bar{T}=\infty$. In particular, (19) holds for all time $t \geq t_{0}$ and can be equivalently written as

$$
\begin{array}{r}
\alpha\left(\left|\varphi_{\xi}(t)-\varphi_{\zeta}(t)\right|\right) \leq \max \left\{\beta\left(\left|\varphi_{\xi}\left(t_{0}\right)-\varphi_{\zeta}\left(t_{0}\right)\right|, t-t_{0}\right) ;\right. \\
\left.\kappa\left(\left\|\varphi_{\xi}-\varphi_{\zeta}\right\|_{\left[t_{0}-\theta ; t\right]}\right)\right\} . \quad(21)
\end{array}
$$

Consequently, considering $t_{0}+\left(t-t_{0}\right) / 2=\left(t+t_{0}\right) / 2$ as the initial time in this equation ensures that

$$
\begin{aligned}
& \alpha\left(\left|\varphi_{\xi}(t)-\varphi_{\zeta}(t)\right|\right) \leq \\
& \max \left\{\beta\left(\left|\varphi_{\xi}\left(\left(t+t_{0}\right) / 2\right)-\varphi_{\zeta}\left(\left(t+t_{0}\right) / 2\right)\right|,\left(t-t_{0}\right) / 2\right) ;\right. \\
& \left.\quad \kappa\left(\left\|\varphi_{\xi}-\varphi_{\zeta}\right\|_{\left[t_{0}+\left(t-t_{0}\right) / 2-\theta ; t\right]}\right)\right\} .
\end{aligned}
$$

In view of (20), this gives

$$
\begin{aligned}
\alpha\left(\left|\varphi_{\xi}(t)-\varphi_{\zeta}(t)\right|\right) \leq \max \{ & \beta\left(\sigma_{0}\left(\|\xi-\zeta\|_{\left[t_{0}-\theta ; t_{0}\right]}\right), \frac{t-t_{0}}{2}\right) ; \\
& \left.\kappa\left(\left\|\varphi_{\xi}-\varphi_{\zeta}\right\|_{\left[t_{0}+\left(t-t_{0}\right) / 2-\theta ; t\right]}\right)\right\} .
\end{aligned}
$$

In the case when $t \geq t_{0}+4 \theta$, the above bound ensures that

$$
\begin{aligned}
\alpha\left(\left|\varphi_{\xi}(t)-\varphi_{\zeta}(t)\right|\right) \leq & \max \left\{\beta\left(\sigma_{0}\left(\|\xi-\zeta\|_{\left[t_{0}-\theta ; t_{0}\right]}\right), \frac{t-t_{0}}{2}\right) ;\right. \\
& \left.\kappa\left(\left\|\varphi_{\xi}-\varphi_{\zeta}\right\|_{\left[t_{0}+\left(t-t_{0}\right) / 4 ; t\right]}\right)\right\} .
\end{aligned}
$$

Now, consider the function defined for all $s, t \in \mathbb{R}_{\geq 0}$ by

$$
\bar{\beta}(s, t):=\max \left\{\sigma_{0}(s) e^{1-t / 4 \theta} ; \beta\left(\sigma_{0}(s), t / 2\right)\right\} .
$$

Recalling that $\sigma_{0} \in \mathcal{K}$, it can be seen that the function $\bar{\beta}$ is a $\mathcal{K} \mathcal{L}$ function and it holds for all $s \in \mathbb{R}_{\geq 0}$ that

$$
\begin{aligned}
& \bar{\beta}(s, t) \geq \beta\left(\sigma_{0}(s), t / 2\right), \quad \forall t \geq 0 \\
& \bar{\beta}(s, t) \geq \sigma_{0}(s), \quad \forall t \leq 4 \theta .
\end{aligned}
$$

Based on this and (22), we obtain that for all $t \geq t_{0}$,

$$
\begin{aligned}
\alpha\left(\left|\varphi_{\xi}(t)-\varphi_{\zeta}(t)\right|\right) \leq & \max \left\{\bar{\beta}\left(\|\xi-\zeta\|_{\left[t_{0}-\theta ; t_{0}\right]}, t-t_{0}\right)\right. \\
& \left.\kappa\left(\left\|\varphi_{\xi}-\varphi_{\zeta}\right\|_{\left[t_{0}+\left(t-t_{0}\right) / 4 ; t\right]}\right)\right\} .
\end{aligned}
$$

We claim that this ensures that $\lim _{t \rightarrow \infty}\left|\varphi_{\xi}(t)-\varphi_{\zeta}(t)\right|=$ 0 . To see this assume the contrary, that is: $\lim \sup _{t \rightarrow \infty}\left|\varphi_{\xi}(t)-\varphi_{\zeta}(t)\right|>0$. In view of (20), this quantity would necessarily be finite: in other words, there would exist $\varepsilon>0$ such that

$$
\limsup _{t \rightarrow \infty}\left|\varphi_{\xi}(t)-\varphi_{\zeta}(t)\right|=\varepsilon .
$$

In particular, there would exist an increasing time sequence $\left\{t_{k}\right\}_{k \in \mathbb{N}}$, satisfying $\lim _{k \rightarrow+\infty} t_{k}=+\infty$, such that

$$
\lim _{k \rightarrow \infty}\left|\varphi_{\xi}\left(t_{k}\right)-\varphi_{\zeta}\left(t_{k}\right)\right|=\varepsilon .
$$

Consequently, it would also hold that

$$
\limsup _{k \rightarrow \infty}\left\|\varphi_{\xi}-\varphi_{\zeta}\right\|_{\left[t_{0}+\left(t_{k}-t_{0}\right) / 4 ; t_{k}\right]} \geq \varepsilon .
$$

In view of (26), it would necessarily follow that

$$
\limsup _{k \rightarrow \infty}\left\|\varphi_{\xi}-\varphi_{\zeta}\right\|_{\left[t_{0}+\left(t_{k}-t_{0}\right) / 4 ; t_{k}\right]}=\varepsilon .
$$

This together with (25) and the fact that $\bar{\beta} \in \mathcal{K} \mathcal{L}$ would then imply that $\alpha(\varepsilon)=\kappa(\varepsilon)$, which contradicts the small-gain condition (11). We conclude that, as claimed,

$$
\lim _{t \rightarrow \infty}\left|\varphi_{\xi}(t)-\varphi_{\zeta}(t)\right|=0 .
$$

Since the above reasoning holds uniformly in $t_{0}$, we conclude that, given any $\xi, \zeta \in \mathcal{C}\left(\left[t_{0}-\theta, t_{0}\right]\right)^{n}$, there exists a function $\eta_{\xi, \zeta} \in \mathcal{L}$ such that

$$
\left|\varphi_{\xi}(t)-\varphi_{\zeta}(t)\right| \leq \eta_{\xi, \zeta}\left(t-t_{0}\right), \quad \forall t \geq t_{0} .
$$

Considering any function $\bar{\eta}: \mathbb{R}_{\geq 0} \times \mathbb{R}_{\geq 0} \rightarrow \mathbb{R}_{\geq 0}$ satisfying

$$
\bar{\eta}(s, t) \geq \max _{\|\xi-\zeta\| \leq s} \eta_{\xi, \zeta}(t), \quad \forall s, t \geq 0,
$$


let the function $\hat{\beta}$ defined as

$$
\hat{\beta}(s, t):=\min \left\{\sigma_{0}(s) ; \bar{\eta}(s, t)\right\}, \quad \forall s, t \geq 0 .
$$

In view of (25), the function $\bar{\eta}(\cdot, t)$ can be picked as a continuous function for all $t \in \mathbb{R}_{>0}$. It follows that $\hat{\beta} \in \mathcal{K} \mathcal{L}$ and it holds from (25) that, for all $t \geq t_{0}$,

$$
\left|\varphi_{\xi}(t)-\varphi_{\zeta}(t)\right| \leq \hat{\beta}\left(\left\|\varphi_{\xi}-\varphi_{\zeta}\right\|_{\left[t_{0}-\theta ; t_{0}\right]}, t-t_{0}\right),
$$

which concludes the proof.

\section{Proof of Proposition 1}

The proof is itself based on the following lemma, which follows along the lines of [31, Lemma 2]. It establishes the existence of a forward and backward complete solutions for delayed systems admitting a forward invariant set.

Lemma 3: Let $M \subset \mathbb{R}^{n}$ be a forward invariant compact set for system (1). Then there is a solution to (1) that evolves in $M$ and is defined for all times $t \in \mathbb{R}$.

Proof: [Sketch] Let $\mathcal{M}$ denote the set of all functions of $\mathcal{C}([-\theta ; 0])^{n}$ taking value in $M$. For each $k \in \mathbb{N}$, let $F_{k}:=\bigcup_{\xi \in \mathcal{M}} \varphi(0 ;-k, \xi)$. Clearly, we have that $F_{0} \subset M$. In addition, it holds that $F_{k+1} \subset F_{k}$ for all $k \in \mathbb{N}$. It follows in particular that $F_{k} \subset M$ for all $k \in \mathbb{N}$. Furthermore, the sets $F_{k}$ being all closed and nested, the set $\bigcap_{k>0} F_{k}$ is nonempty. Following the reasoning in the proof of [31, Lemma 2], we conclude that there exists a solution $x(\cdot)$ that is defined for all times $t \in \mathbb{R}$, satisfying $\phi(0) \in \bigcap_{k \in \mathbb{N}} F_{k}$, and by construction taking values only in $M$.

Now, assume that the system (1) is uniformly incrementally stable. Invoking Lemma 3, it admits a solution $x(\cdot)$ which is bounded and defined for all times. It then follows from Definition 1 that there exists $\beta \in \mathcal{K} \mathcal{L}$ such that, for all $t_{0} \in \mathbb{R}$ and all $\xi \in \mathcal{C}\left(\left[t_{0}-\theta: t_{0}\right]\right)^{n}$,

$$
\left|\varphi\left(t ; t_{0}, \xi\right)-x(t)\right| \leq \beta\left(\|\xi-x\|_{\left[t_{0}-\theta ; t_{0}\right]}, t-t_{0}\right), \quad \forall t \geq t_{0},
$$

which establishes uniform convergence.

Conversely, if (1) is uniformly convergent on a compact set $M$, then it is also uniformly incrementally stable on $M$ : this follows by a reasoning very similar to that employed in [26, proof of Theorem 8].

\section{Proof of Proposition 2}

In view of Proposition 1, the system (1) is uniformly convergent. We therefore proceed as in the proof of [20, Property 2.23]. Let $\bar{x}: \mathbb{R} \rightarrow \mathbb{R}^{n}$ be the bounded steady-state solution of Definition 2, and let $\tilde{x}$ be defined as $\tilde{x}(t):=\bar{x}(t+T)$ for all $t \in \mathbb{R}$. Then $\tilde{x}$ is a solution of

$$
\dot{x}(t)=f\left(t+T, x,(x)_{t}\right)=f\left(t, x,(x)_{t}\right),
$$

where the second equality comes from the $T$-periodicity of the vector field $f$. Consequently, $\bar{x}$ and $\tilde{x}$ are two solutions of (1) that are bounded over $\mathbb{R}$. However, invoking ${ }^{1}[20$, Property 2.15], the steady-state solution of a uniformly convergent system is unique. This shows that $\tilde{x}(t)=\bar{x}(t+T)=\bar{x}(t)$ for all $t \in \mathbb{R}$, meaning that the signal $\bar{x}$ to which all solutions converge is a $T$-periodic signal.

\footnotetext{
${ }^{1}$ That result is actually stated for non-delayed systems, but it extends straightforwardly to time-delayed systems.
}

\section{E. Proof of Proposition 3}

To establish Proposition 3, we make use of Theorem 1. To that aim, consider the non-delayed system associated to (12):

$$
\dot{x}(t)=A x(t)+g(t, v),
$$

where $v \in \mathcal{C}([-\theta ;+\infty))^{n}$ denotes an exogenous input. Let $V(x, y):=(x-y)^{T} P(x-y)$ for all $x, y \in \mathbb{R}^{n}$, where the matrix $P \in \mathbb{R}^{n \times n}$ satisfies Assumption 1. Then (3) holds with

$$
\underline{\alpha}(s)=p_{m} s^{2}, \quad \bar{\alpha}(s)=p_{M} s^{2}, \quad \forall s \in \mathbb{R}_{\geq 0},
$$

and straightforward computations yield for all $v, w \in$ $\mathcal{C}([-\theta ;+\infty))^{n}$ and all $t \in \mathbb{R}_{\geq 0}$,

$$
\begin{aligned}
\dot{V}:= & \frac{\partial V}{\partial x}(A x+g(t, v))+\frac{\partial V}{\partial y}(A y+g(t, w)) \\
= & (x-y)^{T}\left[A^{T} P+P A\right](x-y) \\
& +2(x-y)^{T} P[g(t, v)-g(t, w)] .
\end{aligned}
$$

In view of Assumption 1, this gives

$\dot{V} \leq-(x-y)^{T} Q(x-y)+2 p_{M}|x-y||g(t, v)-g(t, w)|$.

We now invoke the following result, which can be found in [9, Chapter X-6].

Lemma 4: [9] For any matrices $P, Q \in \mathbb{R}^{n \times n}$, with $Q$ positive definite, it holds that

$$
\min \{\lambda \in \mathbb{R}: \operatorname{det}(P-\lambda Q)=0\}=\min _{x \in \mathbb{R}^{n}} \frac{x^{T} P x}{x^{T} Q x} .
$$

With this lemma, it follows from (14) and (30) that

$$
\dot{V} \leq-\frac{1}{\lambda_{0}} V+2 p_{M}|x-y||g(t, v)-g(t, w)| .
$$

Moreover, it holds that

$$
|g(t, v)-g(t, w)|=\left[\sum_{i=1}^{n}\left|g_{i}(t, v)-g_{i}(t, w)\right|^{2}\right]^{1 / 2}
$$

with

$$
\begin{aligned}
\left|g_{i}(t, v)-g_{i}(t, w)\right|= & S_{i}\left(u_{i}(t)+\sum_{j=1}^{n} c_{i j} v_{j}\left(t-\delta_{i j}\right)\right) \\
& -S_{i}\left(u_{i}(t)+\sum_{j=1}^{n} c_{i j} w_{j}\left(t-\delta_{i j}\right)\right) \mid .
\end{aligned}
$$

Exploiting Assumption 2, this gives

$$
\begin{aligned}
\left|g_{i}(t, v)-g_{i}(t, w)\right| & \leq \sigma_{i}\left|\sum_{j=1}^{n} c_{i j}\left(v_{j}\left(t-\delta_{i j}\right)-w_{j}\left(t-\delta_{i j}\right)\right)\right| \\
& \leq \sigma_{i}\|v-w\| \sum_{j=1}^{n}\left|c_{i j}\right| .
\end{aligned}
$$

Plugging this into (32) provides the following bound:

$$
|g(t, v)-g(t, w)| \leq \ell\|v-w\|,
$$

where

$$
\ell:=\sqrt{\sum_{i=1}^{n}\left(\sigma_{i} \sum_{j=1}^{n}\left|c_{i j}\right|\right)^{2}}
$$


Going back to (31), it follows that

$$
\dot{V} \leq-\frac{1}{\lambda_{0}} V+2 p_{M} \ell|x-y|\|v-w\|
$$

Now, from (15) and (33), there exists $\epsilon>0$ such that

$$
\kappa_{0}:=\frac{\left(2 p_{M} \lambda_{0} \ell(1+\epsilon)\right)^{2}}{p_{m}}<p_{m} .
$$

With this constant, straightforward computations lead to

$$
V \geq \kappa_{0}\|v-w\|^{2} \Rightarrow \dot{V} \leq-\frac{\epsilon}{\lambda_{0}(1+\epsilon)} V
$$

which makes (4) satisfied with $\rho(s)=\epsilon p_{m} s^{2} / \lambda_{0}(1+\epsilon)$ and

$$
\kappa(s)=\kappa_{0} s^{2}
$$

for all $s \in \mathbb{R}_{\geq 0}$. In view of (34), it holds that $\kappa_{0}<p_{m}$ which, in view of (29) and (35) make the small-gain condition (5) satisfied. Theorem 1 thus guarantees that (12) is uniformly incrementally stable.

Finally, assume that the functions $S_{i}$ are bounded, and let $\bar{S}>0$ be any constant satisfying $\left|S_{i}(r)\right| \leq \bar{S}$ for all $i \in\{1, \ldots, n\}$. Consider the Lyapunov function candidate $W(x):=x^{T} P x$. Then, in view of Assumption 1 it holds that

$$
\dot{W} \leq-q_{m}|x|^{2}+2 \sqrt{n} p_{M} \bar{S} \leq-\frac{q_{m}}{p_{M}} W(x)+2 \sqrt{n} p_{M} \bar{S}
$$

Consequently, it holds that

$$
W(x) \geq \frac{2 \sqrt{n} p_{M}^{2} \bar{S}}{q_{m}} \Rightarrow \dot{W} \leq 0,
$$

which ensures the forward invariance of the compact set

$$
M=\left\{x \in \mathbb{R}^{n}: W(x) \leq \frac{2 \sqrt{n} p_{M}^{2} \bar{S}}{q_{m}}\right\} .
$$

The fact that, in response to any $T$-periodic (resp. constant) input $u$ the state $x$ asymptotically converges to a $T$-periodic (resp. constant) solution is then a direct consequence of Proposition 2.

\section{CONCLUSION AND PERSPECTIVES}

We have adapted the Razumikhin-Lyapunov approach to provide conditions under which nonlinear delayed systems are incrementally stable. The considered class of systems includes time-varying dynamics, thus allowing to consider the influence of exogenous inputs (as long as the incremental stability holds uniformly in the input signal). It encompasses systems with multiple, possibly time-varying, bounded delays. We also deduced conditions under which the limit solution of such a system is $T$-periodic when the considered input is itself $T$ periodic. These results have been applied to Lur'e systems with multiple delays, which constitutes a promising soil for the analysis of pathological oscillations in the firing-rate of neuronal populations.

\section{REFERENCES}

[1] N. Aghannan and P. Rouchon. An intrinsic observer for a class of lagrangian systems. IEEE Trans. on Automat. Contr., 48(6):936-945, 2003.

[2] M.A. Aizerman and F.R. Gantmacher. Absolute Stability of Regulator Systems. Holden-Day, 1964.

[3] D. Angeli. A Lyapunov approach to incremental stability properties. IEEE Trans. on Automat. Contr., 47(3):410-421, 2002.

[4] S.J. Chung and J.J. Slotine. Cooperative robot control and concurrent synchronization of lagrangian systems. IEEE Transactions on Robotics, 25(3):686-700, 2009.

[5] S.J. Chung and J.J. Slotine. On synchronization of coupled HopfKuramoto oscillators with phase delays. In Proc. 49th. IEEE Conf. Decision Contr., pages 3181-3187, 2010.

[6] B. Demidovich. Lectures on Stability Theory. in Russian, Moscow, 1967.

[7] C.A. Desoer and M. Vidyasagar. Feedback Systems: Input-Output Properties. Academic Press, New York, 1975.

[8] F. Forni and R. Sepulchre. A differential Lyapunov framework for contraction analysis. Submitted to IEEE Trans. on Automatic Control, 2013. Preprint avalailable at: http://arxiv.org/abs/1208.2943.

[9] F. R. Gantmacher. The theory of matrices, vols. I and II. Chelsea publishing Co., New York, 1959.

[10] H. Haidar, W. Pasillas-Lépine, E. Panteley, and A. Chaillet. Basal ganglia oscillations: the role of delays and external excitatory nuclei. In To appear in Proc. European Control Conference, Zurich, Switzerland, 2013.

[11] J.K. Hale and S.M.V. Lunel. Introduction to functional differential equations, volume 99. Springer, 1993.

[12] C. Hammond, H. Bergman, and P. Brown. Pathological synchronization in Parkinson's disease: networks, models and treatments. Trends in Neurosciences, 30(7):357 - 364, July 2007. INMED/TINS special issue-Physiogenic and pathogenic oscillations: the beauty and the beast.

[13] Q. Han. Absolute stability of time-delay systems with sector-bounded nonlinearity. Automatica, 41(12):2171-2176, December 2005.

[14] A.J. Nevado Holgado, J.R. Terry, and R. Bogacz. Conditions for the Generation of Beta Oscillations in the Subthalamic Nucleus-Globus Pallidus Network. Journal of Neuroscience, 30(37):12340, 2010.

[15] B. Jayawardhana, H. Logemann, and E. Ryan. The Circle Criterion and Input-to-State Stability. IEEE Control Systems, 31(4):32-67, 2011.

[16] H. Khalil. Nonlinear systems. Macmillan Publishing Co., 2nd ed., New York, 1996.

[17] W. Lohmiller and J.J. Slotine. On contraction analysis for nonlinear systems. Automatica, 34(6), 1998.

[18] W. Michiels and S.I Niculescu. Stability and Stabilization of TimeDelay Systems: An Eigenvalue-Based Approach. Society for Industrial Mathematics (Advances in Design and Control), 2008.

[19] W. Pasillas-Lépine. Delay-induced oscillations in Wilson and Cowan's model: An analysis of the subthalamo-pallidal feedback loop in healthy and parkinsonian subjects. To appear in Biological Cybernetics, February 2013.

[20] A.V. Pavlov, N. van de Wouw, and H. Nijmeijer. Uniform Output Regulation of Nonlinear Systems: A Convergent Dynamics Approach. Systems \& Control: Foundations \& Applications, 2006.

[21] A.V. Pavlov, N. van de Wouw, and H. Nijmeijer. Frequency response functions for nonlinear convergent systems. IEEE Trans. on Automat. Contr., 52(6):1159-1165, 2007.

[22] V. Pliss. Nonlocal Problems of the Theory of Oscillations. Academic Press London, 1966.

[23] G. Pola, P. Pepe, M. Di Benedetto, and P. Tabuada. Symbolic models for nonlinear time-delay systems using approximate bisimulations. Systems \& Control Letters, 59(6):365-373, 2010.

[24] V. Rasvan, D. Danciu, and D. Popescu. Frequency domain stability inequalities for nonlinear time delay systems. In MELECON 2010 15th IEEE Mediterranean Electrotechnical Conference, pages 13981401,2010

[25] B.S. Razumikhin. Application of Liapunov's method to problems in the stability of systems with a delay. Avtomat. i Telemeh, 21:740-774, 1960.

[26] B.S. Rüffer, N. van de Wouw, and M. Mueller. Convergent systems vs. incremental stability. Syst. \& Contr. Letters, 62:277-285, 2013.

[27] G. Russo, M. di Bernardo, and E.D. Sontag. Global entrainment of transciptional systems to periodic inputs. PLoS Computational Biology, 6(4):1-26, 2010. 
[28] E. Steur and H. Nijmeijer. Synchronization in Networks of Diffusively Time-Delay Coupled (Semi-)Passive Systems. IEEE Trans. on Automat. Contr., 58(6):1358-1371, 2011.

[29] A.R. Teel. Connections between Razumikhin-type theorems and the ISS nonlinear small gain theorem. IEEE Trans. on Automat. Contr., 43(7):960-964, 1998.

[30] S. Tiwari, Y. Wang, and Z.P. Jiang. Remarks on integral-ISS for systems with delays. Intelligent Control and Automation (WCICA), 2012.

[31] V.A. Yakubovich. The matrix-inequality method in the theory of the stability of nonlinear control systems. I: The absolute stability of forced vibrations. Autom. Rem. Control, 25:905-917, 1965. 